\title{
ERRATUM
}

F. R. Scarano - H. M. Duarte - A. C. Franco •

A. Geßler • E. A. de Mattos • H. Rennenberg •

U. Lüttge

\section{Physiological synecology of tree species in relation to geographic distribution and ecophysiological parameters at the Atlantic forest periphery in Brazil: an overview}

Published online: 10 January 2006

(C) Springer-Verlag 2006

Trees (2005) 19: 493-496

In the above article the year 2004 in the source line is wrong, it should read: Trees (2005) 19:493-496.

The online version of the original article can be found at http://dx.doi.org/10.1007/s00468-005-0420-9

F. R. Scarano $(\square) \cdot$ E. A. de Mattos

Departamento de Ecologia, Universidade Federal do Rio de Janeiro, CCS, IB,

Caixa Postal 68020, cep 21941-970 Rio de Janeiro, Brazil

e-mail: fscarano@biologia.ufrj.br

H. M. Duarte · U. Lüttge

Institut für Botanik, Darmstadt University of Technology,

Schnittspahnstr. 3-5,

64287 Darmstadt, Germany

A. C. Franco

Departamento de Botânica, Universidade de Brasília,

Caixa Postal 04457, cep 70919-970 Brasília, Brazil

A. Geßler $\cdot$ H. Rennenberg

Institut für Forstbotanik und Baumphysiologie, Freiburg

University,

Georges-Köhler Allee 53/54,

79085 Freiburg, Germany 